\title{
Intérêt de la valeur propre des descendances S1 associée à la valeur en croisement avec un testeur pour la sélection du maïs fourrage
}

\author{
J.-P. Sampoux, A. Gallais et M. Lefort-Buson \\ INRA, CNRS, UPS, Ferme du Moulon, 91190 Gif-sur-Yvette, France
}

(reçu le 13 juin 1988, accepté le 7 mars 1989)

Résumé - Dans le cadre d'un cycle de sélection récurrente appliqué à quatre populations de maïs fourrage, les descendances $\mathrm{S} 1$ et les descendances en croisement avec un testeur hybride simple des plantes candidates à la sélection ont été récoltées en plante entière. Pour chaque population, certains des caractères suivants ont été observés en cours de végétation sur les deux types de descendances : vigueur de départ, date de floraison, hauteur totale et hauteur de l'épi, longueur et largeur de la feuille de l'épi.

La dépression entre descendances en croisement et descendances S1 affecte plus fortement le rendement en plante entière et les caractères de hauteur que la longueur de la feuille de l'épi. Les variabilités génétique et environnementale se montrent plus importantes dans les descendances $\mathbf{S 1}$ que dans les descendances en croisement pour le rendement et les caractères de morphologie. De fortes corrélations génétiques sont toujours observées entre les deux types de descendances pour les caractères de précocité. La corrélation entre les deux types de descendances est, par contre, très variable selon les populations étudiées pour le rendement en plante entière (ou les caractères de hauteur). Lorsqu'un progrès génétique est souhaitable sur les deux types de descendances, il est donc préférable de sélectionner sur un index des critères de valeur en croisement et de valeur propre des descendances S1. Pour l'amélioration du rendement en plante entière des descendances en croisement, la prise en considération de critères de valeur propre des descendances $\mathrm{S} 1$ est au moins aussi efficace que l'observation de caractères associés sur les descendances en croisement.

Zea mays - sélection récurrente - sélection multicaractère - index de sélection - héritabilité - progrès génétique

Summary - S1 value combined with topcross value for forage maize selection. The study concerned four forage maize populations, improved following recurrent selection schemes. Selfed (S1) and topcross progenies with a single cross tester were tested for whole-plant silage yield early. The following traits were also studied for the two kinds of progenies : early vigour, early flowering, plant and ear heights, length and width of ear leaf.

The depression between topcross and S1 progenies was larger for whole-plant yield and height traits than for ear leaf length. Genetic and environmental variability for yield and morphological traits were broader for $S 1$ progenies than for topcross progenies. Genetic correlations between earliness traits (early flowering or dry matter content) of S1 families and topcross progenies were always high.

Genetic correlations between yield (or height traits) of S1 families and topcross progenies were not similar in the four populations. So index breeding taking into account topcross and S1 criteria seemed the best way for the improvement of both $S 1$ value and cross value of populations. For the improvement of topcrosses whole-plant yield, taking into account $S 1$ value criteria was at least as efficient as studying topcross associated traits.

Zea mays - recurrent selection - multitrait selection - selection index - heritability - genetic gain

\section{Introduction}

A court terme, la sélection récurrente pour l'aptitude à la combinaison avec un testeur à base étroite est une méthode d'amélioration des populations préparant bien à la création de variétés hybrides. Elle peut en effet se prolonger facilement par la sélection généalogique sur la valeur en combinaison avec le testeur utilisé pour l'amélioration de la population (Gallais, 1978). Dans de tels programmes de sélection récurrente, une sélection sur les descendances issues d'une autofécondation des plantes testées pour la valeur en croisement (descendances S1) peut également être envisagée avec deux objectifs : 1. améliorer la valeur propre des lignées fixées 
dérivables de la population, afin de produire plus facilement des hybrides simples; 2 . mieux apprécier la valeur génétique en croisement (Gallais, 1977).

L'étude qui suit a été réalisée dans le cadre de la sélection récurrente de populations de maïs précoce, améliorées pour la valeur en croisement avec un testeur hybride simple, selon des critères de valeur fourragère (récolte en plante entière). Elie a pour but de préciser l'intérêt d'une sélection sur descendances $\mathrm{S} 1$ pour de tels programmes.

\section{Matériel et Méthodes}

\section{Matériel}

Les résultats ont été obtenus sur les tests d'un premier cycle de sélection récurrente de populations de maïs précoce. Ces populations sont issues d'intercroisements de synthétiques de lignées, préalablement améliorées par sélection récurrente sur la valeur fourragère à la station d'amélioration des plantes de Lusignan (Tableau I). Les testeurs des populations cornées (SRC84 et SRC85) et dentées (SRD85) ont été choisis de façon à exploiter la vigueur hybride existant entre les groupes de combinaison corné (d'origine européenne) et denté (d'origine nord-américaine). Les testeurs corné et denté sont, de plus, apparentés aux populations respectivement cornées et dentées, de façon à développer au mieux la complémentarité entre ces deux groupes de populations. La population SRCD85 a été produite en intercroisant une population cornée et une population dentée dans le but d'accumuler dans une même population le maximum de gènes favorables (Gallais, 1978). Un premier cycle de sélection récurrente sur testeur hybride simple cornédenté, dont les tests de descendances sont inclus dans l'étude présentée, a été réalisé de façon à restreindre cette population au matériel montrant une bonne aptitude à la combinaison avec chacun des deux groupes classiques (deux sélections divergentes, sur testeur corné et sur testeur denté, seront amorcées par la suite).

Pour toutes les populations étudiées, un cycle de trois ans a été choisi. En première année, les plantes So de chaque population ont été autofécondées et croisées avec le testeur. En deuxième année, les descendances en croisement des plantes $\mathrm{S} 0$ et une partie des descendances $\mathrm{S} 1$ ont été installées en essais récoltés en plante entière. En troisième année, les descendances $\mathrm{S} 1$ des plantes S0 sélectionnées ont été intercroisées pour produire la population du cycle de sélection suivant.

Les tests de seconde année ont été réalisés à la ferme du Moulon, en 1984 pour la population SRC84, et en 1985 pour les populations SRC85, SRD85 et SRCD85. Le dispositif expérimental de chaque population était composé de plusieurs lattices à deux répétitions (Tableau II). Les parcelles, constituées chacune d'une ligne de $5 \mathrm{~m}$ et espacées de $0,80 \mathrm{~m}$, avaient été semées avec les densités suivantes : 95000 plantes/ha pour les descendances en croisement, 75000 plantes/ha en 1984 et 95000 plantes/ha en 1985 pour les descendances $S 1$.

Tableau I. Populations étudiées et leurs testeurs.

Population

Dénomination

Groupe de combinaison des lignées constitutives
Testeur

Formule Groupe de combinaison

\begin{tabular}{|c|c|c|c|c|}
\hline SRC85 & cornées & $\mathrm{F} 250$ & F252 & denté \\
\hline SRD85 & dentées & $\mathrm{F} 2$ & F251 & corné \\
\hline SRCD85 & cornées et dentées & F251 & F252 & corné-denté \\
\hline SRC84 & cornées & F250 & F252 & denté \\
\hline
\end{tabular}

Tableau II. Protocoles expérimentaux.

Descendances en croisement

Population
Nombre de descendances
Nombre d'essais

\section{Dispositif} lattice
Descendances S1

\begin{tabular}{llccccc} 
Population & $\begin{array}{l}\text { Nombre de } \\
\text { descendances }\end{array}$ & $\begin{array}{l}\text { Nombre } \\
\text { d'essais }\end{array}$ & $\begin{array}{l}\text { Dispositif } \\
\text { lattice }\end{array}$ & $\begin{array}{l}\text { Nombre de } \\
\text { descendances }\end{array}$ & $\begin{array}{l}\text { Nombre } \\
\text { d'essais }\end{array}$ & $\begin{array}{l}\text { Dispositif } \\
\text { lattice }\end{array}$ \\
\hline SRC85 & 156 & 3 & $7 \times 8$ & 56 & 1 & $7 \times 8$ \\
SRD85 & 156 & 3 & $7 \times 8$ & 56 & 1 & $7 \times 8$ \\
SRCD85 & 208 & 4 & $7 \times 8$ & 56 & 1 & $7 \times 8$ \\
SRC84 & 204 & 3 & $8 \times 9$ & 81 & 1 & $9 \times 9$ \\
\hline
\end{tabular}

2 répétitions pour tous les essais. 


\section{Caractères observés}

Différents caractères ont été observés sur les deux types de descendances. La vigueur de départ $(v d)$ est appréciée par une notation visuelle selon une échelle de 1 (faible) à 9 (vigoureux) réalisée au stade 7-9 feuilles visibles. La date de floraison femelle (dflo) correspond à la date à laquelle $50 \%$ des soies sont apparues. Certains caractères de morphologie sont observés après la floraison sur un échantillon de cinq plantes par parcelle : la hauteur totale $(h t)$ du sol à la base de la première ramification de la panicule, la hauteur de l'épi supérieur (he) du sol au nœud de l'épi, la longueur de la feuille de l'épi supérieur (long), et sa largeur (larg) mesurée dans la zone la plus large. Les essais d'un type de descendances d'une population, groupés, sont récoltés en une même journée pour un niveau de teneur en matière sèche compris entre 25 et $30 \%$. Pour chaque parcelle, la matière verte totale est pesée. Le rendement sec en plante entière $(r d t)$ est calculé après estimation de la teneur en matière sèche (\% ms) par séchage à l'étuve d'un échantillon de matière verte de $800 \mathrm{~g}$.

Des notations de sensibilité à la verse (verse racinaire et courbure des tiges) avaient également été réalisées sur les deux types de descendances. Elles n'ont pas été retenues dans cette étude, car elles conduisent souvent à des variances résiduelles importantes. Une sélection par niveaux indépendants, à partir des notations de verse et d'un index des autres caractères, semble un bon compromis tant que de meilleurs critères de résistance à la verse n'auront pas été définis.

\section{Interprétation biométrique des tests de seconde année}

Pour chaque population, la variation liée aux caractères a été décomposée dans tous les essais selon le modèle suivant: $Y_{i j k}=\mu+G_{i}+b_{j}+s b_{j k}+E_{i j k}$, où $Y_{i j k}$ est la performance phénotypique du génotype i placé dans le $k^{e}$ sous-bloc du bloc $j ; \mu$ est la moyenne générale de la population $j$; $G$ et $E$ sont des variables aléatoires liées au génotype et à l'erreur, $b$ et $s b$ étant des effets fixes dus aux blocs et aux sous-blocs. La structure en lattice n'a pas été prise en compte.

Pour les descendances en croisement de chaque population, l'absence d'hétérogénéité nette entre les différents essais (I) a permis une estimation globale des matrices de somme de produits associées aux effets phénotypiques $\left(\mathbf{S P}_{\mathrm{P}}=\Sigma_{/} \mathrm{SP}^{\prime} \mathrm{p}\right)$ et aux effets résiduels $\left(\mathbf{S P}_{W}=\Sigma_{l} \mathbf{S P}_{W}{ }_{W}\right)$, avec une sommation des degrés de liberté. Les estimations des composantes génétiques $\Sigma_{\mathrm{G}}$ et résiduelles $\Sigma_{\mathrm{W}}$ de la variance phénotypique ont été obtenues selon la méthode de Henderson III (Searle, 1971), d'une part pour les descendances en croisement, et d'autre part pour les descendances $\mathrm{S} 1$.

Par ailleurs, les covariances résiduelles entre caractères observés sur des types de descendances différents étant, par construction, d'espérance nulle, les covariances phénotypiques entre ces caractères sont égales aux covariances génétiques.

La meilleure estimation de la valeur génétique d'un caractère, tenant compte des relations entre différents caractères observés, est obtenue par régression génophénotypique (Henderson, 1975; Vincourt et Gallais, 1983). Le degré de précision de l'estimation de la valeur génétique pour certains caractères sera estimé par le carré de la corrélation entre valeur génétique vraie et valeur estimée $\left[r^{2}(G, G)\right]$ pour une sélection univariable (héritabilité univariable), et pour l'observation de plusieurs caractères (héritabilité multivariable). Dans le cas des descendances en croisement, les coefficients $\left[r^{2}(G, G)\right]$ sont également calculés pour le cas théorique de quatre répétitions, ce calcul étant réalisé à partir des matrices de variance - covariances génétique et résiduelle estimées sur deux répétitions. Différents index des estimateurs des caractères principaux seront construits. Les pondérations économiques seront déterminées en fonction des progrès génétiques souhaités (Rouvier, 1977). L'estimation de la différence entre la moyenne des familles sélectionnées et la moyenne générale de la population est également une estimation du progrès génétique attendu après intercroisement, sauf dans le cas de la prévision du progrès génétique sur la valeur propre des descendances $\mathrm{S} 1$, lorsque des caractères $\mathrm{S} 1$ sont observés. La variance génétique entre familles $S 1$ comprend en effet un terme de dominance (Gallais, 1989), et la différence entre moyenne des familles sélectionnées et moyenne générale surestime alors le progrès génétique prévisible. Les progrès génétiques seront estimés pour une intensité de sélection de $20 \%$.

\section{Résultats}

\section{Etude préliminaire sur les moyennes des popu-} lations

Pour les caractères de morphologie et le rendement en plante entière, la valeur moyenne des descendances $\mathrm{S} 1$ est nettement inférieure à celle des descendances en croisement, quelle que soit la population (Tableau III). Cette dépression entre croisements et $\mathrm{S} 1$ affecte moins la longueur de la feuille de l'épi $(4,4 \%$ à $9,7 \%)$ que le rendement en plante entière $(10,5 \%$ à $35,4 \%)$ et les hauteurs totale et de l'épi ( $13,1 \%$ à $37,7 \%)$.

Ecarts-types génétiques et résiduels, héritabilités univariables

\section{Descendances en croisement avec le testeur}

Les héritabilités univariables (Tableau IV) les plus fortes sont, en général, observées pour les caractères de précocité (date de floraison, teneur en matière sèche) et pour la longueur de la feuille de l'épi. La hauteur totale et celle de l'épi présentent aussi une héritabilité forte, mais moins élevée que celle des caractères précédents. Des héritabilités plus faibles et plus variables, selon les populations, sont observées pour le rendement en plante entière. Ces résultats sont cohérents avec ceux de Gallais et al. (1983) obtenus sur des matériels génétiques apparentés.

\section{Descendances S1}

Les héritabilités univariables des descendances S1 (Tableau IV) sont toujours nettement plus élevées que celles des descendances en croise- 
Tableau III. Performances moyennes des populations SRC85(a), SRD85(b), SRCD85(c) et SRC84(d).

\begin{tabular}{|c|c|c|c|c|c|c|c|c|}
\hline \multirow[b]{2}{*}{$\begin{array}{l}\text { Moyenne des descendances } \\
\text { en croisement }\end{array}$} & \multicolumn{2}{|c|}{$\begin{array}{l}\text { Hauteur totale } \\
\text { ht }(\mathrm{cm})\end{array}$} & \multicolumn{2}{|c|}{$\begin{array}{l}\text { Hauteur épi } \\
\text { he }(\mathrm{cm})\end{array}$} & \multicolumn{2}{|c|}{$\begin{array}{l}\text { Longueur feuille } \\
\text { long }(\mathrm{cm})\end{array}$} & \multicolumn{2}{|c|}{$\begin{array}{l}\text { Rendement } \\
\text { rdt } \\
\text { (tonnes/ha) }\end{array}$} \\
\hline & $\begin{array}{l}181,7(\mathrm{a}) \\
176,1(\mathrm{~b})\end{array}$ & $\begin{array}{l}169,0(c) \\
184,1(d)\end{array}$ & $\begin{array}{l}88,0 \\
86,2\end{array}$ & $\begin{array}{l}79,6 \\
81,9\end{array}$ & $\begin{array}{l}70,7 \\
71,9\end{array}$ & $\begin{array}{l}67,5 \\
72,6\end{array}$ & $\begin{array}{l}14,8 \\
14,3\end{array}$ & $\begin{array}{l}14,3 \\
13,8\end{array}$ \\
\hline $\begin{array}{l}\text { Moyenne des descendances } \\
\text { S1 }\end{array}$ & $\begin{array}{l}130,7 \\
139,1\end{array}$ & $\begin{array}{l}146,9 \\
134,5\end{array}$ & $\begin{array}{l}59,0 \\
63,8\end{array}$ & $\begin{array}{l}69,0 \\
51,0\end{array}$ & $\begin{array}{l}64,5 \\
64,9\end{array}$ & $\begin{array}{c}64,5 \\
-\end{array}$ & $\begin{array}{l}10,6 \\
12,5\end{array}$ & $\begin{array}{r}12,8 \\
8,9\end{array}$ \\
\hline $\begin{array}{l}\text { Dépression entre croisements } \\
\text { et } S 1\left(^{*}\right)\end{array}$ & $\begin{array}{l}28,1 \\
21,0\end{array}$ & $\begin{array}{l}13,1 \\
26,9\end{array}$ & $\begin{array}{l}32,9 \\
25,9\end{array}$ & $\begin{array}{l}13,3 \\
37,7\end{array}$ & $\begin{array}{l}8,8 \\
9,7\end{array}$ & $\begin{array}{l}4,4 \\
-\end{array}$ & $\begin{array}{l}28,4 \\
12,6\end{array}$ & $\begin{array}{l}10,5 \\
35,4\end{array}$ \\
\hline
\end{tabular}

Tableau IV. Ecarts-types génétiques $\left(\sigma_{G}\right)$, résiduels $\left(\sigma_{W}\right)$, et héritabilités univariables $\left(h^{2} u\right)$ estimés pour les descendances en croisement avec le testeur (tc) et pour les descendances S1 (S1).

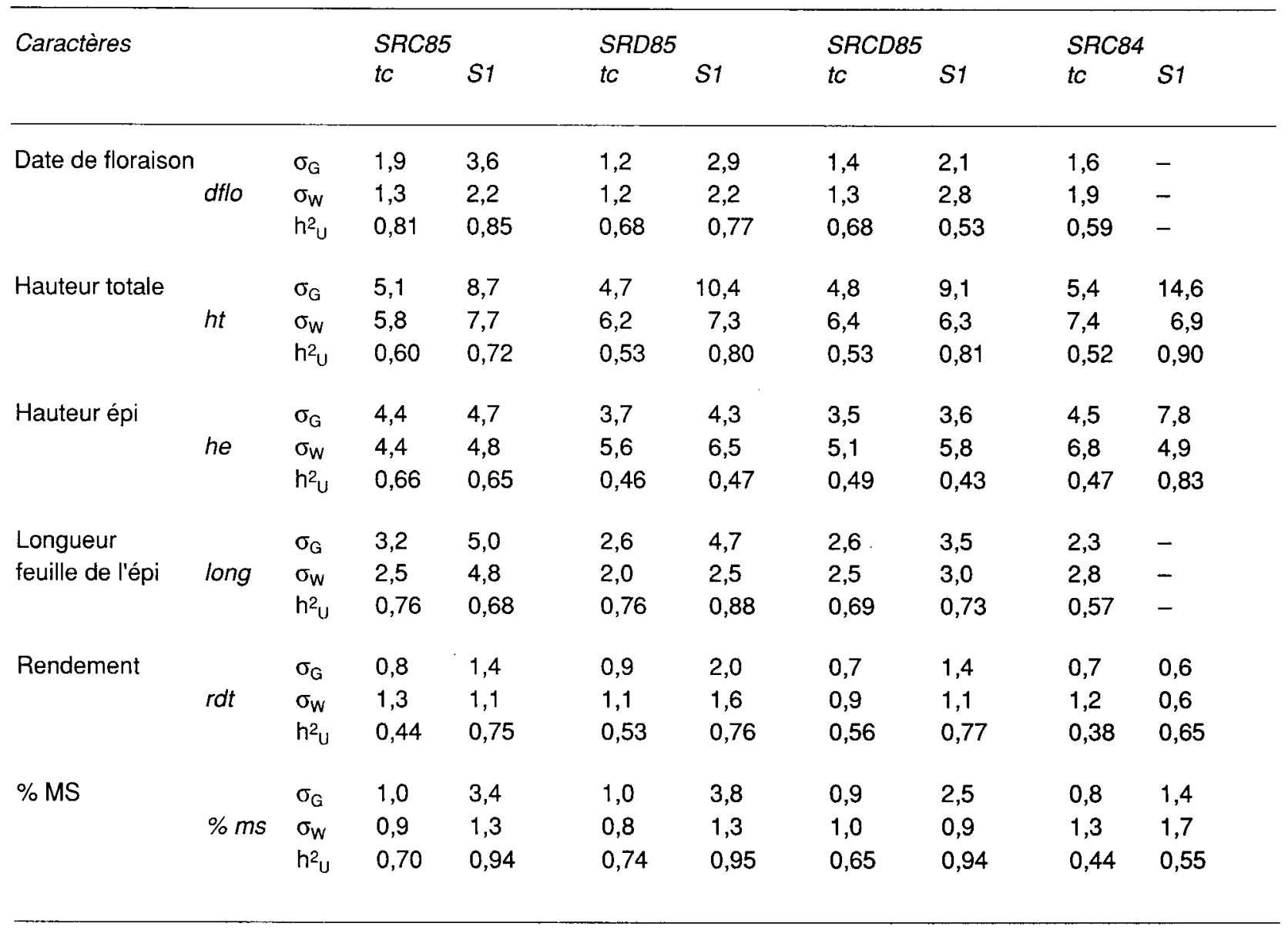

$\mathrm{h}_{\mathrm{U}}^{2}=\sigma_{\mathrm{G}}^{2} /\left(\sigma_{\mathrm{G}}^{2}+\frac{1}{N} \sigma_{W}^{2}\right)$, pour $\mathrm{N}=2$

Ordre de grandeur des intervalles de confiance à $5 \%$ de quelques valeurs de $h^{2} \mathrm{u}$ pour 156 descendances tc et 56 descendances $\$ 1$ (Knapp, 1985).

$h^{2} \mathrm{u} \quad h^{2} u-\alpha_{1}$ (tc) $\quad h^{2}{ }_{u}+\alpha_{2}$ (tc) $\quad h^{2}{ }_{u}-\alpha_{1}$ (S1) $\quad h^{2}{ }_{u}+\alpha_{2}$ (S1)

$\begin{array}{lllll}0,85 & 0,80 & 0,90 & 0,75 & 0,90 \\ 0,65 & 0,50 & 0,75 & 0,40 & 0,80 \\ 0,45 & 0,25 & 0,60 & 0,10 & 0,65\end{array}$


ment pour certains caractères : rendement en plante entière, teneur en matière sèche, hauteur totale des plantes. Les autres caractères présentent, en général, des héritabilités du même ordre de grandeur pour les deux types de descendances.

Les héritabilités plus fortes en S1 qu'en croisement sont liées, dans la plupart des cas, à une variance génétique plus importante dans les descendances $S 1$, comme cela pouvait être attendu d'un point de vue théorique (Gallais, 1989), alors que la variance résiduelle reste du même ordre de grandeur dans les deux types de descendances. D'autre part, pour l'ensemble des caractères de morphologie et pour le rendement en plante entière, les coefficients de variation génétique et résiduelle (écart-type rapporté à la moyenne) sont plus élevés sur les descendances S1 que sur les descendances en croisement, ce qui illustre bien la plus grande variabilité génétique des descendances $\mathrm{S} 1$, mais aussi leur moindre homéostasie.

\section{Corrélations génétiques}

- Entre caractères observés sur les descendances en croisement

Des corrélations génétiques élevées (Tableau $\mathrm{V}$ ) apparaissent, quelle que soit la population, entre le rendement en plante entière et les caractères de morphologie, ainsi qu'entre la date de floraison et la teneur en matière sèche. Elles confirment notamment l'intérêt des plantes de gabarit important pour l'amélioration du rendement en plante entière ainsi que la possibilité de prédire partiellement la précocité de récolte (\% ms tc) par la précocité de floraison. Les corrélations génétiques entre rendement en plante entière (ou caractères de morphologie) et caractères de précocité (date de floraison, teneur en matière sèche) sont par contre assez variables d'une population à l'autre. Elles ne font, en particulier, ressortir une liaison négative entre rendement en plante entière et précocité, que dans le cas des populations cornées (SRC84 et SRC85). Ces résultats sont cohérents avec ceux déjà signalés par Gallais et al. (1983) sur des matériels génétiques apparentés.

- Entre caractères observés sur les descendances $S 1$

Sur les descendances $S 1$, la variabilité génétique semble être organisée de façon sensiblement différente de ce qui a été observé sur les descendances en croisement. Dans l'ensemble, les corrélations génétiques (Tableau $V$ ) entre rendement en plante entière et caractères de morphologie sont en effet plus faibles que sur les descendances en croisement. De plus, sur les descendances $S 1$ des populations cornées, le rendement en plante entière n'est plus corrélé avec la teneur en matière sèche ou la date de floraison.

\section{- Entre caractères observés sur des types de descendances différents}

Les valeurs de ces corrélations (Tableau VI), témoignent aussi d'une modification de l'expression de la variabilité génétique avec le type de descendance. Pour les caractères sensibles à la dépression entre croisements et $\mathrm{S} 1$, comme le rendement et les caractères de hauteur, la corrélation entre les deux types de descendances est très variable selon les populations. Elle est par

Tableau V. Corrélations génétiques $\left(r_{\mathrm{G}}\right)$ entre caractères observés sur les descendances en croisement d'une part, et entre caractères observés sur les descendances $S 1$ d'autre part.

\begin{tabular}{|c|c|c|c|c|c|c|c|c|c|c|c|c|}
\hline \multirow[b]{2}{*}{ rdt tc } & \multicolumn{2}{|c|}{$v d$ tc } & \multicolumn{2}{|c|}{ dflo tc } & \multicolumn{2}{|c|}{ ht tc } & \multicolumn{2}{|c|}{ he tc } & \multicolumn{2}{|c|}{ long tc } & \multicolumn{2}{|c|}{$\% \mathrm{~ms} \mathrm{tc}$} \\
\hline & $\begin{array}{l}-0,08(a) \\
-0,17(b)\end{array}$ & $\begin{array}{r}0,20(d) \\
(d)\end{array}$ & $\begin{array}{l}0,53 \\
0,23\end{array}$ & $\begin{array}{l}0,05 \\
0,43\end{array}$ & $\begin{array}{l}0,72 \\
0,52\end{array}$ & $\begin{array}{l}0,63 \\
0,54\end{array}$ & $\begin{array}{l}0,76 \\
0,54\end{array}$ & $\begin{array}{l}0,48 \\
0,77\end{array}$ & $\begin{array}{l}0,50 \\
0,42\end{array}$ & $\begin{array}{l}0,76 \\
0,58\end{array}$ & $\begin{array}{r}-0,43 \\
0,18\end{array}$ & $\begin{array}{l}-0,22 \\
-0,52\end{array}$ \\
\hline \multirow[t]{2}{*}{$\% m s \quad t c$} & $\begin{array}{l}0,11 \\
0,28\end{array}$ & $\begin{array}{c}0,00 \\
-\end{array}$ & $\begin{array}{l}-0,71 \\
-0,64\end{array}$ & $\begin{array}{l}-0,54 \\
-0,71\end{array}$ & $\begin{array}{l}-0,25 \\
-0,24\end{array}$ & $\begin{array}{l}-0,01 \\
-0,49\end{array}$ & $\begin{array}{l}-0,25 \\
-0,05\end{array}$ & $\begin{array}{r}0,03 \\
-0,65\end{array}$ & $\begin{array}{l}-0,17 \\
-0,25\end{array}$ & $\begin{array}{l}-0,31 \\
-0,27\end{array}$ & & \\
\hline & \multicolumn{2}{|c|}{$v d S 1$} & \multicolumn{2}{|c|}{ dflo S1 } & \multicolumn{2}{|c|}{ ht $\mathrm{S} 1$} & \multicolumn{2}{|c|}{ he $S 1$. } & \multicolumn{2}{|c|}{ long S1 } & \multicolumn{2}{|c|}{$\% m s \mathrm{~s} 1$} \\
\hline rdt $S 1$ & $\begin{array}{l}0,39 \\
0,60\end{array}$ & - & $\begin{array}{r}-0,01 \\
0,17\end{array}$ & $\begin{array}{l}0,21 \\
-\end{array}$ & $\begin{array}{l}0,45 \\
0,60\end{array}$ & $\begin{array}{l}0,37 \\
0,46\end{array}$ & $\begin{array}{l}0,25 \\
0,73\end{array}$ & $\begin{array}{l}0,04 \\
0,42\end{array}$ & $\begin{array}{r}-0,05 \\
0,23\end{array}$ & $\begin{array}{c}0,39 \\
-\end{array}$ & $\begin{array}{l}0,18 \\
0,10\end{array}$ & $\begin{array}{r}0,20 \\
-0,09\end{array}$ \\
\hline$\% m s \quad s 1$ & $\begin{array}{l}0,25 \\
0,39\end{array}$ & - & $\begin{array}{l}-0,82 \\
-0,84\end{array}$ & $\begin{array}{c}-0,62 \\
-\end{array}$ & $\begin{array}{r}-0,17 \\
0,06\end{array}$ & $\begin{array}{r}0,03 \\
-0,32\end{array}$ & $\begin{array}{l}0,03 \\
0,28\end{array}$ & $\begin{array}{r}0,06 \\
-0,57\end{array}$ & $\begin{array}{l}-0,81 \\
-0,75\end{array}$ & $\begin{array}{c}-0,29 \\
-\end{array}$ & & \\
\hline
\end{tabular}

Populations : SRC85 (a), SRD85 (b), SRCD85 (c), SRC84 (d)

Erreurs standard (Scheinberg, 1966):

- croisements : $0,10 \leq$ S.E. $\left(r_{G}\right) \leq 0,20$

$-\mathrm{S} 1: 0,10 \leq \mathrm{S}$.E. $\left(r_{\mathrm{G}}\right) \leq 0,25$. 
Tableau VI. Corrélations génétiques entre caractères observés sur les descendances en croisement (tc) et caractères observés sur les descendances S1 (S1).

\begin{tabular}{|c|c|c|c|c|c|c|c|c|c|c|c|c|c|c|c|}
\hline \multirow[b]{2}{*}{$r d t$} & \multirow[b]{2}{*}{ tc } & \multicolumn{2}{|c|}{$v d S 1$} & \multicolumn{2}{|c|}{ dffo $S 1$} & \multicolumn{2}{|c|}{$h t S 1$} & \multicolumn{2}{|c|}{ he $S 1$} & \multicolumn{2}{|c|}{ long $S 1$} & \multicolumn{2}{|c|}{$r d t S 1$} & \multicolumn{2}{|c|}{$\% m s \quad S 1$} \\
\hline & & $\begin{array}{l}0,42(a \\
0,00(b\end{array}$ & $\begin{array}{l}-(c) \\
-(d)\end{array}$ & $\begin{array}{l}0,39 \\
0,56\end{array}$ & $\begin{array}{c}-0,09 \\
-\end{array}$ & $\begin{array}{l}0,19 \\
0,21\end{array}$ & $\begin{array}{l}0,22 \\
0,72\end{array}$ & $\begin{array}{l}0,24 \\
0,03\end{array}$ & $\begin{array}{l}0,18 \\
0,72\end{array}$ & $\begin{array}{l}0,64 \\
0,61\end{array}$ & $\begin{array}{c}0,41 \\
-\end{array}$ & $\begin{array}{l}0,34 \\
0,31\end{array}$ & $\begin{array}{c}0,11 \\
(1,00)\end{array}$ & $\begin{array}{l}-0,48 \\
-0,14\end{array}$ & $\begin{array}{l}-0,40 \\
-0,52\end{array}$ \\
\hline$\% m s$ & tc & $\begin{array}{l}0,31 \\
0,26\end{array}$ & - & $\begin{array}{l}-0,69 \\
-0,63\end{array}$ & $\begin{array}{c}(-1,00) \\
-\end{array}$ & $\begin{array}{l}0,28 \\
0,06\end{array}$ & $\begin{array}{r}0,18 \\
-0,01\end{array}$ & $\begin{array}{l}0,61 \\
0,06\end{array}$ & $\begin{array}{l}-0,11 \\
-0,04\end{array}$ & $\begin{array}{l}-0,50 \\
-0,33\end{array}$ & $\begin{array}{c}-0,46 \\
-\end{array}$ & $\begin{array}{l}-0,05 \\
-0,14\end{array}$ & $\begin{array}{r}-0,13 \\
0,39\end{array}$ & $\begin{array}{l}0,72 \\
0,56\end{array}$ & $\begin{array}{l}0,49 \\
0,60\end{array}$ \\
\hline$\left({ }^{*}\right)$ & tc & $\begin{array}{l}0,18 \\
0,21\end{array}$ & $\begin{array}{l}- \\
-\end{array}$ & $\begin{array}{l}0,97 \\
0,86\end{array}$ & $\begin{array}{l}0,86 \\
-\end{array}$ & $\begin{array}{l}0,19 \\
0,37\end{array}$ & $\begin{array}{l}0,92 \\
0,95\end{array}$ & $\begin{array}{l}0,58 \\
0,64\end{array}$ & $\begin{array}{l}0,25 \\
0,65\end{array}$ & $\begin{array}{l}0,86 \\
0,60\end{array}$ & $(1,00)$ & & & & \\
\hline
\end{tabular}

(") tc : corrélation entre un caractère S1 et le même caractère observé en croisement

Populations : SRC85 (a), SRD85 (b), SRCD85 (c), SRC84 (d)

contre toujours élevée pour la longueur de la feuille de l'épi, peu affectée par la consanguinité.

La longueur de la feuille de l'épi apparaît, d'autre part, comme le caractère des descendances $\mathrm{S} 1$ le mieux corrélé au rendement des descendances en croisement; mais elle est également corrélée négativement avec leur teneur en matière sèche. Le rendement et les caractères de hauteur des descendances $\mathrm{S} 1$ ne sont bien corrélés au rendement des descendances en croisement que dans le seul cas de la population SRC84.

Pour l'ensemble des populations, de bonnes corrélations sont notées entre caractères de précocité des deux types de descendances (date de floraison et teneur en matière sèche).

Différents auteurs ont également étudié la corrélation entre descendances en croisement et descendances $\mathrm{S} 1$ pour des caractères observés sur des populations améliorées pour le rendement en grain (Genter et Alexander, 1962; Lonnquist et Lindsey, 1964; Carangal et al., 1971; Harris et al., 1972). En ce qui concerne l'humidité du grain, des corrélations fortes sont toujours observées entre les deux types de descendances. Ceci est cohérent avec les corrélations génétiques toujours élevées obtenues sur les populations étudiées entre caractères de précocité des deux types de descendances. Des valeurs très différentes sont, par contre, observées pour la corrélation entre rendements en grain des deux types de descendances. Ceci peut s'expliquer par l'hétérogénéité des matériels étudiés (nature et ampleur de la variabilité génétique), mais également, comme l'avaient constaté Harris et al. (1972), par l'influence des conditions environnementales. Ce dernier facteur pourrait en partie expliquer que la forte corrélation génétique entre rendements en plante entière des deux types de descendances, observée pour la population SRC84, ne se retrouve pas sur les populations expérimentées en 1985 (en particulier sur la population SRC85 proche par sa composition génétique de la population SRC84).

Sélection sur les descendances en croisement et réponses corrélatives sur les descendances S1

Le rendement en plante entière des descendances en croisement est un caractère peu facile à sélectionner. Son héritabilité est, en effet, souvent assez médiocre. Une sélection plus efficace sur ce caractère peut, en général, être réalisée à partir d'une estimation multivariable prenant en compte l'information apportée par la teneur en matière sèche et par différents caractères observés en cours de végétation sur les descendances en croisement (caractères de morphologie, date de floraison femelle) (Gallais et al., 1983). Pour toutes les populations étudiées, de telles estimations multivariables conduisent effectivement à une meilleure efficacité de la sélection sur le rendement des descendances en croisement. L'observation de caractères en cours de végétation apporte un gain de précision équivalant à la récolte de une (SRC84) à deux répétitions supplémentaires (populations expérimentées en 1985) (Tableau VII). Sauf pour la population SRC84, l'héritabilité de la teneur en matière sèche est plus élevée que celle du rendement en plante entière. Une estimation multivariable peut toutefois être également efficace sur ce caractère (Tableau VII).

Les conséquences d'une sélection réalisée sur deux répétitions des descendances en croisement ont donc été étudiées pour le choix sur un index des valeurs génétiques du rendement et de la teneur en matière sèche, estimées en tenant compte des observations réalisées en cours de végétation. Les coefficients de l'index de chaque population ont été choisis de façon à obtenir un maintien du niveau de précocité 
Tableau VIl. Comparaison de la précision de l'estimation de la valeur génétique $\left(r^{2}(G, G)\right)$ des caractères principaux des descendances en croisement pour différents dispositifs expérimentaux : sélection sur les descendances en croisement seules avec 2 ou 4 répétitions, ou bien sélection combinée sur 2 répétitions des descendances en croisement plus 2 répétitions des descendances $S 1$.

\begin{tabular}{|c|c|c|c|c|c|}
\hline \multirow{2}{*}{ Dispositif expérimental } & \multirow{3}{*}{$\begin{array}{l}\text { Caractères prédicteurs } \\
\text { Sélection univariable directe }\end{array}$} & \multicolumn{4}{|c|}{$r(G, \hat{G})$} \\
\hline & & \multicolumn{2}{|c|}{$r d t$ tc } & \multicolumn{2}{|c|}{$\% m s t c$} \\
\hline 2 répétitions des & & $0,44(a)$ & $0,56(c)$ & 0,70 & 0,65 \\
\hline descendances & & $0,53(b)$ & 0,38 (d) & 0,74 & 0,44 \\
\hline \multirow[t]{4}{*}{ en croisement } & A & 0,57 & 0,61 & 0,78 & 0,70 \\
\hline & & 0,53 & 0,53 & 0,75 & 0,58 \\
\hline & $\mathrm{B}$ & 0,70 & 0,72 & 0,84 & 0,71 \\
\hline & & 0,60 & 0,59 & 0,76 & 0,61 \\
\hline \multirow{4}{*}{$\begin{array}{l}4 \text { répétitions des descendances } \\
\text { en croisement }\end{array}$} & $A$ & 0,68 & 0,74 & 0,87 & 0,81 \\
\hline & & 0,70 & 0,66 & 0,86 & 0,71 \\
\hline & B & 0,79 & 0,81 & 0,91 & 0,82 \\
\hline & & 0,74 & 0,71 & 0,86 & 0,73 \\
\hline \multirow{10}{*}{$\begin{array}{l}2 \text { répétitions des descendances } \\
\text { en croisement } \\
+ \\
2 \text { répétitions des descendances } \\
S 1\end{array}$} & C & 0,62 & 0,62 & 0,81 & 0,71 \\
\hline & & 0,56 & $(1,00)$ & 0,77 & 0,80 \\
\hline & $\mathrm{D}$ & 0,68 & 0,63 & 0,89 & 0,72 \\
\hline & & 0,65 & 0,76 & 0,77 & 0,60 \\
\hline & $E$ & 0,76 & 0,90 & 0,82 & 0,86 \\
\hline & & 0,65 & - & 0,77 & - \\
\hline & $\mathrm{F}$ & 0,72 & 0,80 & 0,90 & 0,81 \\
\hline & & 0,63 & - & 0,77 & - \\
\hline & $\mathrm{G}$ & 0,69 & 0,83 & 0,83 & 0,73 \\
\hline & & 0,67 & - & 0,78 & - \\
\hline
\end{tabular}

Populations : SRC85 (a), SRD85 (b), SRCD85 (c), SRC84 (d)

Les dispositifs expérimentaux étudiés sont comparés pour l'observation de différentes séries de caractères prédicteurs :

- A : rdt tc, \% ms tc

$-\mathrm{B}: \mathrm{A}, \mathrm{ht}$ tc, he tc, long tc, dflo tc

$-C: A, r d t S 1, \%$ ms $S 1$

- D : A, ht S1, he S1, long S1, dflo S1, dflo S1 $(a, b, c)$ - $\mathrm{E}: \mathrm{A}$, long tc, long $\mathrm{S} 1$, larg $\mathrm{S} 1$, dflo $\mathrm{S} 1$

$-\mathrm{F}: \mathrm{A}$, long $t c_{1}$ ht $\mathrm{S1}$, he $\mathrm{S1}$, dflo $\mathrm{S1}$

$\mathrm{A}$, ht $\mathrm{S} 1$, he $\mathrm{S} 1$ (d)

(teneur en matière sèche) (Tableau VIII). La réponse du rendement en plante entière des descendances $\mathrm{S} 1$ à une telle sélection n'apparaît pas toujours satisfaisante. Elle est en effet négative (Tableau VIII) pour deux populations (SRC85, SRCD85). Toutefois, ainsi que le souligne l'étude des corrélations entre les deux types de descendances, ces réponses corrélatives pourraient être assez variables selon les conditions environnementales.

Sélection sur les descendances $S 1$ et réponses corrélatives sur les descendances en croisement

La productivité des descendances en croisement (rendement en plante entière) n'est pas toujours bien liée à celle des descendances S1. L'étude des corrélations et de la dépression entre les deux types de descendances laisse cependant supposer que la productivité en croisement puisse être corrélée de façon moins dépendante du matériel génétique, ou de l'environnement, avec certains caractères $S 1$, tels que la longueur de la feuille de l'épi. De plus, les corrélations entre caractères de précocité des deux types de descendances pourraient être toujours assez fortes. Lorsqu'un progrès génétique est souhaité sur la valeur propre des descendances S1 et sur la valeur en croisement, la sélection sur descendances $S 1$ doit donc porter sur un index de caractères liés à la valeur propre des descendances $S 1$ (rendement en plante entière, hauteur des plantes) et de caractères mieux corrélés à la valeur en croisement (longueur de la feuille de l'épi, caractères de précocité). Les pondérations à apporter à un tel index sont cependant difficiles à déterminer en l'absence d'expérimentation des descendances en croisement. L'information apportée, par exemple, par la double expérimentation des descendances en croisement et des S1 au cycle précédent, risquerait d'être d'un intérêt limité, du fait de l'influence probable des conditions expérimentales sur la valeur des corrélations entre caractères des deux types de descendances. L'étude des conséquences d'une sélection multicaractère sur descendances $\mathrm{S} 1$ a donc été limitée au calcul de la précision des 
Tableau VIII. Sélection sur index. Coefficients économiques et progrès génétiques attendus $\left(I \Delta_{G}\right)$.

\begin{tabular}{|c|c|c|c|c|c|c|c|c|c|c|}
\hline \multirow[b]{2}{*}{ Population } & \multirow{2}{*}{$\begin{array}{c}\text { Dispositif } \\
\text { expérimental }\end{array}$} & \multirow{2}{*}{$\begin{array}{l}\text { Caractères } \\
\text { prédicteurs }\end{array}$} & \multicolumn{5}{|c|}{ Coefficients appliqués aux valeurs génétiques } & \multicolumn{3}{|c|}{$I \Delta_{G}$} \\
\hline & & & $r d t \quad t c$ & $\% m s$ tc & rdt $S 1$ & ht $S 1$ & $\begin{array}{l}\text { rdt TC } \\
\text { (tonnes/ } \\
\text { ha) }\end{array}$ & $\begin{array}{l}\% \text { ms TC } \\
\text { (points) }\end{array}$ & $\begin{array}{l}\text { rot } S 1 \\
\text { (tonnes/ } \\
\text { ha) }\end{array}$ & $\begin{array}{l}\text { ht } S 1 \\
(\mathrm{~cm})\end{array}$ \\
\hline SRC85 & 2 répétitions des & & 1,00 & 0,45 & & & 0,75 & 0,00 & $-0,11$ & 2,6 \\
\hline SRD85 & descendances en & $\mathrm{B}$ & 1,00 & 0,00 & & & 0,92 & 0,00 & 0,81 & 4,1 \\
\hline SRCD85 & croisement & & 1,00 & 0,35 & & & 0,81 & 0,00 & $-0,15$ & 1,2 \\
\hline SRC84 & & & 1,00 & 0,65 & & & 0,43 & 0,00 & 0,53 & 9,7 \\
\hline SRC85 & & $\mathrm{F}$ & 1,00 & 0,30 & & 0,04 & 0,71 & 0,00 & 0,55 & 7,1 \\
\hline SRD85 & 2 répétitions des & $\mathrm{F}$ & 1,00 & $-0,05$ & & 0,04 & 0,90 & 0,00 & 1,19 & 8,9 \\
\hline SRCD85 & descendances & $\mathrm{F}$ & 1,00 & 0,08 & & 0,05 & 0,81 & 0,00 & 0,14 & 8,1 \\
\hline SRC84 & en croisement & D & 1,00 & 0,54 & & 0,00 & 0,68 & 0,00 & 0,42 & 18,2 \\
\hline SRC85 & + & $\mathrm{G}$ & 1,00 & 0,44 & 0,00 & & 0,75 & 0,00 & 0,75 & 3,9 \\
\hline SRD85 & 2 répétions des & $G$ & 1,00 & $-0,08$ & $-0,01$ & & 1,01 & 0,00 & 1,01 & 3,9 \\
\hline SRCD85 & descendances S1 & G & 1,00 & 0,39 & 0,29 & & 0,79 & 0,00 & 0,79 & 3,2 \\
\hline SRC84 & & C & $-1,00$ & $-0,66$ & 4,80 & & 0,63 & 0,00 & 0,63 & 9,4 \\
\hline $\begin{array}{l}-\mathrm{A}: r d t t c, \% \\
-\mathrm{B}: \mathrm{A}, h t t c \\
-\mathrm{C}: \mathrm{A}, r d t \mathrm{~s}\end{array}$ & $\begin{array}{l}\text { ns tc } \\
\% \text { tc, long tc, dflo tc } \mathrm{S} 1\end{array}$ & & $\begin{array}{l}-D: A \\
-F: A \\
-G: C\end{array}$ & $\begin{array}{l}\text { S1, he } \mathrm{S1} \\
\text { tc, ht S1, } \\
\text { tc, long } \mathrm{S}\end{array}$ & $S 1, d f l o$ & & & & & \\
\hline
\end{tabular}

estimations multivariables obtenues avec différents protocoles d'observation des descendances $\mathrm{S} 1$ : observation de caractères en cours de végétation, récolte en plante entière complétée ou non par l'observation de caractères en cours de végétation. Les héritabilités multivariables obtenues pour le rendement et la teneur en matière sèche des descendances en croisement sont en général peu satisfaisantes (Tableau IX). Des valeurs proches de l'héritabilité univariable ne sont obtenues simultanément pour ces deux caractères que sur la population SRC85, avec la mesure de caractères en cours de végétation.

Sélection simultanée sur les deux types de descendances

\section{- Amélioration de la valeur en croisement}

Recherche d'une meilleure efficacité de la sélection sur le rendement en plantes entières des descendances en croisement Dans le cas de la population SRC84, la mise en expérimentation des descendances $S 1$, au côté des descendances en croisement, renforce de façon très nette l'efficacité de la sélection sur le rendement des descendances en croisement (Tableau VII). L'observation des descendances S1 (récolte en plante entière ou mesure de caractère de hauteur) procure un gain de précision sur l'estimation du rendement en croisement plus important que la prise en considération de caractères notés en cours de végétation sur les descendances en croisement, même si le nombre de répétitions de ces dernières est doublé.
Pour les populations expérimentées en 1985, la récolte en plante entière des descendances $\mathrm{S} 1$ apporte, par contre, peu d'informations sur le rendement en croisement. L'observation de caractères en cours de végétation sur les descendances $S 1$, en particulier celle de caractères de dimension foliaire, apparaît plus efficace (Tableau VII). La réalisation de l'ensemble des observations en cours de végétation sur les descendances $\mathrm{S} 1$, plutôt que sur les descendances en croisement, n'induit pas de perte de précision sur l'estimation du rendement en croisement des populations SRC85 et SRD85; elle entraîne cependant une moindre efficacité de la sélection sur ce caractère pour la population SRCD85. La mesure d'un caractère de morphologie sur les descendances en croisement, combinée à l'observation de caractères en cours de végétation sur les descendances $S 1$, a procuré les meilleures estimations du rendement en croisement des populations expérimentées en 1985. Sauf pour la population SRD85, ces estimations confèrent une précision équivalente à un doublement du nombre de répétitions des descendances en croisement (Tableau VII).

Estimation de la teneur en matière sèche des descendances en croisement Les caractères observés sur les descendances S1 permettent d'obtenir une estimation de la teneur en matière sèche en croisement, au moins aussi précise que les observations réalisées en cours de végétation sur les descendances en croisement (Tableau VII). Ces estimations multicaractères n'apportent toutefois pas toujours un gain de précision important, par rapport à une estimation 
Tableau IX. Précision ( $\left.r^{2}(G, \hat{G})\right)$ de l'estimation de la valeur génétique des caractères principaux des descendances en croisement prédite par l'observation de caractères des descendances S1.

\begin{tabular}{|c|c|c|c|c|}
\hline \multirow{3}{*}{$\begin{array}{l}\text { Caractères prédicteurs } \\
\begin{array}{l}\text { Sélection univariable directe } \\
\text { (univariate direct selection) }\end{array}\end{array}$} & \multicolumn{4}{|c|}{$r^{2}(G, \hat{G})$} \\
\hline & \multicolumn{2}{|c|}{$r d t t c$} & \multicolumn{2}{|c|}{$\% m s t c$} \\
\hline & $\begin{array}{l}0,44(a) \\
0,53(b)\end{array}$ & $\begin{array}{l}0,56 \text { (c) } \\
0,38 \text { (d) }\end{array}$ & $\begin{array}{l}0,70 \\
0,74\end{array}$ & $\begin{array}{l}0,65 \\
0,44\end{array}$ \\
\hline A & $\begin{array}{l}0,37 \\
0,10\end{array}$ & $\begin{array}{l}0,18 \\
0,98\end{array}$ & $\begin{array}{l}0,49 \\
0,33\end{array}$ & $\begin{array}{l}0,26 \\
0,29\end{array}$ \\
\hline B & $\begin{array}{l}0,76 \\
0,38\end{array}$ & $\begin{array}{l}0,35 \\
0,52\end{array}$ & $\begin{array}{l}0,65 \\
0,34\end{array}$ & $\begin{array}{l}0,64 \\
0,00\end{array}$ \\
\hline $\mathrm{C}$ & $\begin{array}{l}0,76 \\
0,55\end{array}$ & $\begin{array}{c}0,37 \\
(1,00)\end{array}$ & $\begin{array}{l}0,69 \\
0,44\end{array}$ & $\begin{array}{l}0,36 \\
0,29\end{array}$ \\
\hline
\end{tabular}

Populations : SRC85 (a), SRD85 (b), SRCD85 (c), SRC84 (d )

A : rdt $\mathrm{S} 1, \% \mathrm{~ms} \mathrm{~S} 1$

$\mathrm{B}:$ t $\mathrm{S} 1$, long $\mathrm{S} 1$, larg $\mathrm{S} 1$, dflo $\mathrm{S} 1(a, b, c),+$ vd $\mathrm{S} 1(a, b)$

ht $\mathrm{S} 1$, he $\mathrm{S} 1$ (d)

$\mathrm{C}: \mathrm{A}$, ht $\mathrm{S} 1$, long $\mathrm{S} 1$, larg $\mathrm{S} 1(a, b, c),+$ vd $\mathrm{S} 1(a, b)$

A, ht S1, he $\mathrm{S} 1$ (d)

univariable sur deux répétitions des descendances en croisement; une augmentation du nombre de répétitions des descendances en croisement conduit alors à une sélection plus efficace sur ce caractère (Tableau VII).

- Amélioration simultanée de la valeur en croisement et de la valeur propre des descendances $S 1$

Sauf dans le cas de la population SRC84, l'évolution du progrès génétique sur la valeur propre des descendances $S 1$ ne peut être dirigée que si un poids économique est attribué dans la sélection sur index, à un caractère $S 1$, lié à cette valeur propre (rendement en plante entière, ou à défaut hauteur totale des descendances S1). Le choix d'un progrès génétique sur la hauteur totale des descendances $S 1$, au moins égal aux trois quarts de la réponse à la sélection univariable, n'a pas entraîné pour toutes les populations un progrès génétique notable sur le rendement en plante entière des descendances $S 1$ (Tableau VIII). Avec la prise en considération des données de la récolte des descendances $S 1$, une bonne coïncidence entre progrès génétique attendu et progrès génétique souhaité a pu être obtenue pour les critères principaux de valeur en croisement et pour le rendement en plante entière des descendances $S 1$ (un progrès génétique équivalent pour le rendement des descendances en croisement et celui des descendances $\mathrm{S} 1$ a été choisi) (Tableau VIII). Dans le cas de la population SRC84, l'information apportée par les descendances $S 1$ est particulièrement efficace pour une sélection sur index imposant une contrainte sur la précocité de récolte. Le progrès génétique attendu sur le rendement en croisement, avec l'observation des descendances S1, atteint en effet $180 \%$ du progrès prévu par une sélection sur index réalisée sur deux répétitions des descendances en croisement (Tableau VIII).

\section{Discussion - Conclusion}

La mise en expérimentation des descendances S1, au côté des descendances en croisement, apporte un gain de précision sur l'estimation du rendement en croisement au moins équivalent à l'observation de caractères associés en cours de végétation sur les descendances en croisement. Toutefois, ce gain de précision peut rester inférieur à celui procuré par un doublement du nombre de répétitions des descendances en croisement, pratique qui renforcerait, par ailleurs, l'efficacité de la sélection sur la teneur en matière sèche.

Lorsque la sélection ne porte que sur les critères de valeur en croisement, il peut donc être préférable de limiter l'expérimentation aux seules descendances en croisement. Cependant, dans le cas où un progrès génétique est également souhaité sur la valeur propre des descendances $\mathrm{S} 1$, afin de créer des lignées fixées vigoureuses, la réponse corrélative, obtenue par sélection sur les seules descendances en croisement, peut être insuffisante. Un tri préalable sur la biomasse aérienne des descendances $\mathrm{S} 1$ pourrait toutefois entraîner une perte de variabilité préjudiciable aux performances en croisement. Une sélection simultanée sur les deux types de descendances, 
par le choix sur un index de critères de valeur en croisement et de valeur propre des descendances S1, apparaît donc préférable. Dans ce cas, la prise en considération de critères de valeur propre des descendances $\mathrm{S} 1$, en particulier de caractères de morphologie, peut se substituer à l'observation de caractères associés sur les descendances en croisement, tout en maintenant, voire en augmentant, l'efficacité de la sélection sur la valeur en croisement pour le rendement en plante entière.

\section{Références}

Carangal V.R., Ali S.M., Koble A.F., Rinke E.H. \& Sentz J.C. (1971) Comparison of S1 with testcross evaluation for recurrent selection in maize. Crop Sci. 11, 658-661

Gallais A. (1977) Amélioration de l'efficacité des schémas de sélection récurrente. Ann. Amélior. Plant. 27, 477-481

Gallais A. (1978) Amélioration des populations, méthodes de sélection et création de variétés. III. Bases théoriques pour l'étude de la sélection récurrente réciproque. Ann. Amélior. Plant. 28, 637-666

Gallais A., Vincourt P. \& Bertholleau J.C. (1983) Etude de critères de sélection chez le maïs fourrage : hérita- bilités, corrélations génétiques et réponse attendue à la sélection. Agronomie 3(8), 751-760

Gallais A. (1989) Théorie de la Sélection en Amélioration des Plantes. 1 vol. Ed. Masson, Paris

Genter C.F. \& Alexander M.W. (1962) Comparative performance of S1 progenies and test-crosses of corn. Crop Sci. 2, 516-519

Harris R.E., Gardner C.O. \& Compton W.A. (1972) Effects of mass selection and irradiation in corn measured by random $\mathrm{S} 1$ lines and their test-crosses. Crop Sci. 12, 594-598

Henderson C.R. (1975) Best linear unbiased estimation and prediction under a selection model. Biometrics 21, (2), 423-447

Knapp S.J., Stroup W.W. \& Ross W.M. (1985) Exact confidence intervals for heritability on a progeny mean basis. Crop Sc. 25, 192-194

Lonnquist J.H. \& Lindsey M.F. (1964) Top cross versus S1 line performance in corn. Crop Sci. 4, 580-583

Rouvier R. (1977) Mise au point sur le modèle classique d'estimation de la valeur génétique. Ann. Genet. Sel. Anim. 9, 17-26

Scheinberg E. (1966) The sampling variance of the correlation coefficients estimated in genetic experiments. Biometrics 22, 187-191

Searle S.R. (1971) Linear models. 1 vol. Wiley, N.Y.

Vincourt P., Gallais A. (1983) Sur la recherche de critères de sélection : la régression géno-phénotypique. Agronomie 3(9), 827-830 